\title{
Migration Tolerant Human Computer Interaction for Air Traffic Controllers
}

\author{
Oliver Ohneiser and Hejar Gürlük \\ Institute of Flight Guidance, German Aerospace Center (DLR) \\ Lilienthalplatz 7, 38108 Braunschweig, Germany \\ \{Oliver. Ohneiser, Hejar. Guerluek\}@DLR. de
}

\begin{abstract}
Human machine interfaces (HMI) in the product division of air traffic management (ATM) are in use for long time spans. For an efficient use of HMIs not only user centered but also migration tolerant designs are important. Migration tolerance therefore means considering future requirements for a long lasting controller HMI life cycle. For efficient ATM, the concept of system wide information management (SWIM) will be introduced. This generates a large amount of additional information that will influence controller work. In this paper, we therefore describe a new controller role called Information-andConflict-Manager (ICM) who handles the complexity induced by SWIM. The resulting HMI design draft demonstrates how the integration of data could be managed. ICM also supervises training to support controllers successfully passing future flight guidance transitions.
\end{abstract}

Keywords: air traffic control, flight guidance, human machine interaction, migration tolerance, system wide information management, transition steps

\section{Introduction}

The global air traffic is expected to constantly grow, which is also predicted for the future in a range of 1.6 to $3.9 \%$ [1]. In order to cope with this, several challenges like capacity bottlenecks or environmental aspects have to be managed. On the one hand hardware development, i.e. new aircraft avionics and propulsion technologies provide some of the necessary benefits. On the other hand software and methodological solutions are essential to cope with new requirements of future air traffic control, in particular new HMI solutions and flight guidance approaches. As a main actor, air traffic controllers have to be supported in their work. The working environment of air traffic controllers described in chapter 2 encompasses a general radar screen, flight strips, radio telephony and other information tools like weather or aircraft performance data displays. Besides flight strips, the controllers' decisions predominantly are based upon information on the radar screen. In the air traffic controller domain conventional HMIs have life cycles of around 20 years. Work environmental improvements are hardly integrated in terms of HMI updates or enhancements. 
In contrast to new designs in the field of consumer electronics, which appear annually, as for example the smartphone HMIs, the design approach in ATM has to be long-term. Moreover the specifications of ATM research programs, a mix of different capabilities in the aircraft fleet and new information sources require an HMI design tolerant against technological changes and different controller working paradigms.

The research aim of improving ATM processes nowadays is linked with more detailed information about all stakeholders and a more individual planning for all aircraft flights. In the ATM context the system wide information management (SWIM) concept will be introduced, which delivers a variety of data from many different sources building the base of better planning facilities [2]. Indeed better planning is possible but more complex for the responsible planner or controller. Therefore a new working position in allusion to the multi-sector planner [3], [4] called Informationand-Conflict-Manager (ICM) [5] is designed. He monitors incoming information from the SWIM network and forwards only the task-relevant information to the corresponding planner and tactical controller. Additionally he coordinates flight guidance decisions and supervises the training of operational controllers while on duty. The HMI-based training helps controllers to become familiar with the new flight guidance steps (see chapter 2). In conclusion new HMI solutions have to be developed to guarantee communication and coordination between different types of controllers and automation on ground and on board. These interfaces have to come along with small adaptions to the needs of the current guidance step with respect to information, automation and user-centered capabilities [6], [7]. The changing tasks of the controller then can be carried out with the corresponding data of the SWIM network (chapter 3.2) and hence build a basis for a more efficient ATM process.

In section 2 we shortly describe the status quo of controller working positions in air traffic control and their corresponding HMIs. Additionally we highlight the current distance based method of flight guidance and the envisaged transitions towards time based, trajectory based and finally performance based guidance. Section 3 depicts our basic assumptions and requirements for the future air traffic control (ATC) environment and reveals key SWIM elements, which fundamentally contribute to our HMI prototype introduced in chapter 4. Based on requirements for a future ATC environment we will illustrate our concept of an Information-and-Conflict-Manager position and introduce an HMI design layout. Besides that we will also demonstrate the possible HMI solutions of transitions from flight guidance step 1 to another. Section 5 summarizes and concludes our paper.

\section{Background of Air Traffic Management}

Arriving and departing air traffic has to be guided through the terminal maneuvering area (TMA) close to an airport by the responsible air traffic controller. Predefined standard arrival routes and departure routes are used to guide aircraft safely and without collisions. The controller has to ensure safe air traffic from the departure until the arrival. There are different working positions for controllers in the whole ATM process. The en-route controller guides aircraft between airports in large altitudes. 
The arrival, departure and tower controllers handle aircraft in their specific start and end phase or responsibility region. These tactical controllers act more operational than the strategically planner. The conventional flight guidance approach is distance based. To guarantee safety, several rules of the International Civil Aviation Organization (ICAO) have to be followed. The correct separation between different aircraft of e.g. three nautical miles depends on rules in a wake vortex matrix. The controller therefore advises specific flight maneuvers to the pilots. To create more efficient air traffic and enhance existing management, the Single European Sky ATM Research Programme (SESAR) and the US-American project Next Generation Air Traffic Management (NextGen) were founded. The individuality and capabilities of each stakeholder in particular airlines, airports or controller organizations shall be taken more into account for generating improved benefit. Due to SESAR three further flight guidance approach steps will be implemented until a time horizon of 2020. The guidance approach shall be shifted first to a time based (step 1), second to a trajectory based (step 2) and third to a performance based approach (step 3) [8], [9].

Distance based separations between aircraft are used to avoid flights through wake vortexes of other aircraft. But wake vortexes collapse in a certain time not at a certain distance. Hence, in specific wind situations time separation between aircraft would increase capacity. Negotiated times at significant waypoints like runway thresholds or metering fixes become more important. The ground and airborne systems or operators negotiate a certain target time for every waypoint, which at its best ensures collision and wake vortex avoidance with other aircraft at these points. The separation is still important, but the controller can concentrate more on adhering times including separated traffic. Aircraft can fly a more preferred profile as long as negotiated times are kept. Actual HMIs hardly support time based air traffic management. The second step of a trajectory based approach consists of latitudinal, longitudinal, altitudinal and time-related values for every single regular spaced point on the way from an aircraft's actual position to its destination. Beyond negotiated times at significant waypoints, times and other parameter are important at many waypoints on their trajectory. The task of conformance monitoring therefore becomes more important to supervise all aircraft flying along calculated trajectories. This implicitly shall ensure minimal separations and times at significant waypoints. The last step to a performance based flight guidance approach takes into account performance parameter like carbon dioxide emission, kerosene consumption, noise emission and direct operating costs. These aspects are very difficult to handle with current controller HMIs. Furthermore the optima for one aircraft and for the whole air traffic may differ at a certain time. The support by an automated system becomes most important in this step. Separation, negotiated times, trajectories and the environmental and economic parameters have to be supervised. The electronic decision support system should calculate the best options and suggest them to the controller via HMI. The DLR-projects Future Air Ground Integration and flexiGuide have shown some challenges even if reaching step 1 [10] and the need of automation support. Concluding new HMI solutions and especially transitions between HMI evolution states shall support controller work according to the future requirements and methodologies. Additionally a shift of controller working responsibilities can help to alleviate the amount of workload. 


\section{Requirements of Future Air Traffic Control}

This chapter describes basic assumptions for the ATM future and depicts the most important requirements of the system wide information management (SWIM) concept. Based on these requirements we will propose our controller roles and interaction with HMIs.

\subsection{Scenario Assumptions}

Some basic assumptions connected to future development were made to legitimate the following controller and HMI solutions:

- With the growth of world-spanning high-speed telecommunication networks vast data provision and data exchange will be less challenging

- Exact position and navigation surveillance will be delivered

- Communication between controllers and pilots via data link is the predominant way of contact

- There will still be a mix of traffic with aircraft having different equipment degrees like anterior avionics characteristics and capabilities

- All SWIM stakeholders provide their information relevant for others into the network

\subsection{System Wide Information Management}

As described in [2] the concept of SWIM aims at a change in paradigm of how information is managed and spread along the whole (European) ATM system. The implementation of the SWIM concept supports the provision of commonly understood quality information delivered to the ATM stakeholders and therefore spans a great portfolio of information. Although the ATM System covers numerous stakeholders our work will mainly focus on the information supply and exchange between Air Traffic Control and air-side, i.e. approach control and aircraft operators. Given the complex nature of SWIM, which is to go across all ATM systems, data domains, business trajectory phases and the wide range of ATM stakeholders, it is not expected that one solution will fit all. So will neither our solution cover an overall data provision, but it will contribute to a more efficient usage of available data in order to reach the aimed steps of flight guidance (SESAR step 1 to step 3 ) as described in chapter 2. We expect that SWIM therefore will be a key enabler for the future SESAR systems and help to fulfill the envisaged goals as for instance efficiency, optimization of capacity, environmental friendliness and saving economic costs.

In the SESAR work plan, the SWIM concept aims at a paradigm shift from mere message exchange to information publishing, using and contributing, meaning all ATM actors share their information and this requires a more precise and more dynamic way of work [2]. For the realization of our model we propose to take advantage of assumed network technologies in order to enable an efficient information exchange of ATC and aircraft crews resp. airline operation centers. 
For the provision of high-quality and timely information, the so called network operations plan (NOP) of the SWIM concept might be a key tool [12]. The NOP is the central flow management unit web interface for system to system interoperability and has two main benefits. On the one hand it helps monitoring the real-time status of the airspace, the air traffic, its flow and capacity management measures. On the other hand it also supports planning of flight operations in a collaborative way from the strategic to the tactical phases, hence optimizing the use of available ATM capacity. Currently targeted stakeholders are air traffic system reporting offices, aircraft operating centers and flight plan service providers, but we will address the SWIM concept to the air navigation service providers (ANSP) world as well. As mentioned above several data sources are needed for the ANSP in order to serve the flight on several levels such as [13]:

- Flight information - the detailed route of the aircraft defined in four dimensions (4D), so that the position of the aircraft is also defined with respect to the time component

- Aeronautical information - resulting from the assembly, analysis and formatting of aeronautical data

- Meteorological information - on the past, current and future state of earth's atmosphere relevant for air traffic

- Environmental information - about carbon dioxide and kerosene consumption regarding different flight options, and flight areas affected by noise emissions

- Aerodrome Operations information - the status of different aspects of the airport, including approaches, runways, taxiways, gate and aircraft turn-around information

- Capacity and Demand - information on the airspace users' needs of services, access to airspace and airports and the aircraft already using it

- Flow - the network management information necessary to understand the overall air traffic and air traffic services situation

- Surveillance - positioning information from radar, satellite navigation systems, Automatic Dependent Surveillance - Broadcast, aircraft data links, etc.

Not all information needs to be displayed to everyone at any given point of time. The most important data in order to decide quickly and safely have to be shown to the right controller at the right time in the right form with the right workload. Therefore a new controller role will fit this requirement as described in the next chapter.

\section{User-Centered Controller and HMI Design}

An air traffic controllers' working domain has special requirements to guarantee the safe execution of their tasks. This has to be considered when designing an HMI for ATC. The described future requirements have to be handled in future controller working environments. Also in our case the Information-and-Conflict-Manager who manages the information stream of the SWIM network will require a new HMI solution and therefore a new interaction scheme between ICM, controllers and automation. 


\subsection{New Controller Role Information-and-Conflict-Manager}

According to the SWIM concept our approach targets the visualization of SWIM related data obtained by a system-to-system internet communication and the introduction of the Information-and-Conflict-Manager Working (ICM) position. This will be extended in accordance to the flight guidance transition steps (see chapter 2).

The ICM working position is supposed to bridge the data flow between the incoming data sources and the different air traffic controller (ATCO) positions. Besides that, the ICM will handle the traffic on a strategic level. In accordance to new operator roles as proposed in SESAR concepts of operations our ICM embodies in parts the role of the multi-sector-planner (MSP) concept and the dispatcher [11]. The MSP coordinates multiple conventional sectors and plans medium-term aircraft trajectories in his area of responsibility. The balancing of workload for safe flight guidance of all individual sector controllers shall also be guaranteed by the MSP [3]. Similar to the ATCO role of an MSP as proposed in SESAR, the dispatcher role is described [11]. Within this operational concept the dispatcher monitors the traffic in a given airspace, and when a critical event pops up, notified by the system, he/she tries to solve it directly or delegate it to an expert. An expert would be comparable to a tactical controller. The dispatcher therefore is also in charge of managing his/her own workload as well as the workload of the experts by delegating the problems to ATCOs. The dispatcher has a global awareness of the problems and their resolution.

The new operator role represented by the ICM shares both aspects of the MSP and the dispatcher ATCO role but goes a step beyond. He not only manages the strategically planning and conflict resolution of traffic but operates also as a trainer-on-thejob and as an information distributor to the tactical controllers without contacting pilots or making direct operational air traffic management decisions. Complexity is induced by the SWIM network, 4D trajectory management and most important the HMI transitions from step 1 to step 3. Therefore the ICM scans these data and relieves the operational controllers by only forwarding the tactical relevant information. Due to the ICM tasks an HMI for the described requirements is necessary.

Fig. 1 demonstrates a suggestion of a possible prototype of the ICM working position. The concept of the ICM working position proposes an at least 50" display, which is big enough to encompass the several displays at the bottom and the general radar screen as depicted in Fig. 1. The general radar screen displays the overall traffic situation of all supervised tactical controllers within their sector and the universal time coordinated (UTC) in the lower right corner. The radar screen can be used as one integrated display for the available data according to a specific flight. The displays in the bottom bar deliver very different ICM options. If new data concerning one of the nine displays arrives, the corresponding option will be marked. So every incoming data can be noticed. The bottom bar shows SWIM information in all nine displays. The first six displays supplementary allow for communication with stakeholders. 


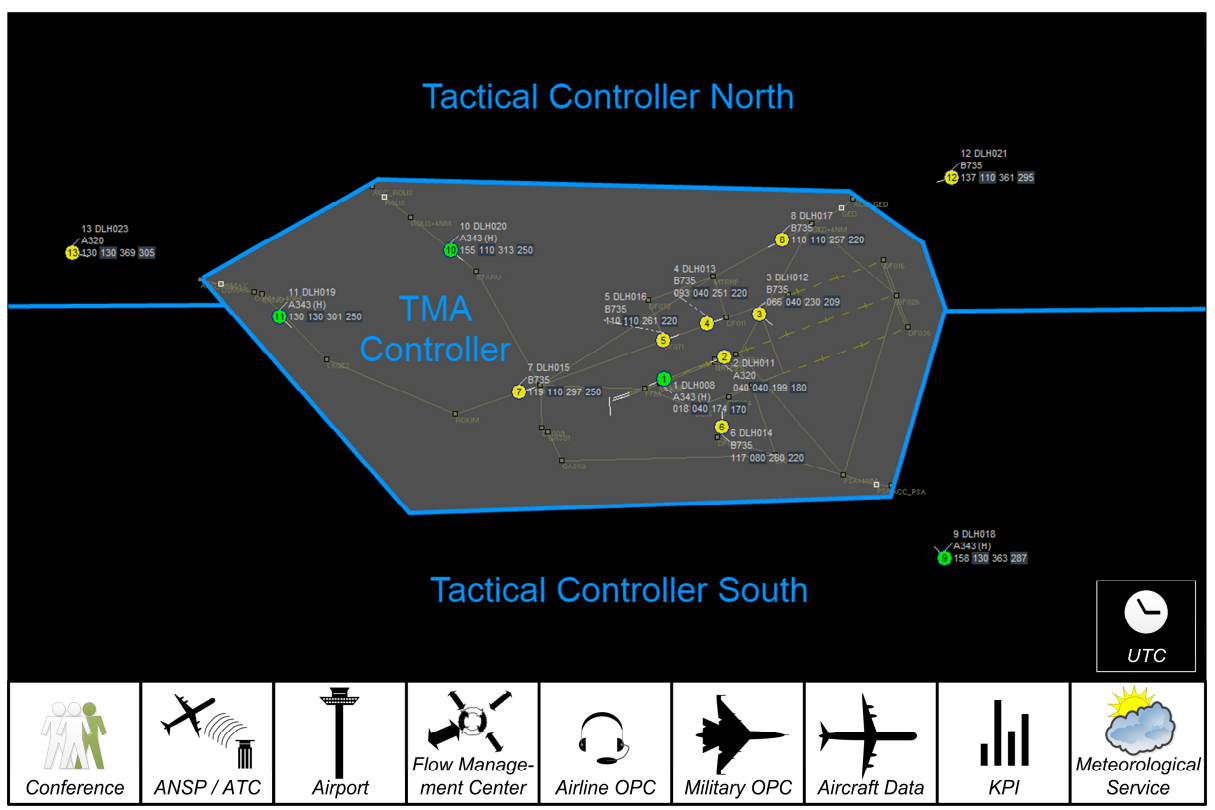

Fig. 1. Display Draft for Information-and-Conflict-Manager Position

The Conference monitor on the outer left enables the communication with the supervised controllers. When selecting an operator position, the ICM awaits a response of the controller. In case of a positive response, the ICM initiates the communication with the corresponding controller via headset. In addition to that, the ICM accesses the controller's radar screen remotely. However, the ICM is not permitted to actively guide the aircraft of the contacted controller.

The ANSP/ATC monitor shows a list of controllers in adjacent regions resp. other ICM positions that can be contacted. The interaction with the different stakeholders could then take place via telephony/voice or text based messages. The Airport display offers a similar functionality including all important airports that can be contacted within neighboring ICM regions. The Airport display contains status information of the selected airport. This includes departures, arrivals, runways in use, aircraft turnaround information and unexpected events.

Via the Flow Management Center display capacity bottlenecks could be identified and solved in advance and communicated to the responsible controller. The Airline Operation Center (OPC) display offers a list of the airlines that are currently in the area of the controllers' responsibility. If necessary the ICM can contact the airlines via headset or by text-messaging. Besides that, flight schedule updates of the controlled flights and actual and planned trajectory information are presented to the ICM. Military OPC display allows for communication about military concerns for flights or airspace restrictions. By selecting an aircraft on the radar screen all corresponding data in the additional displays in the bottom bar are highlighted e.g. airline, airport and ANSP contact and aircraft data. 
The three displays on the right of the bottom bar show Aircraft Data, Key Performance Indicators (KPI) and Meteorological Service data. The aircraft data encompasses minimum and maximum speeds, descent and climb characteristics or actual weight and number of passengers. The KPI display may show ratings about costs, capacity, safety and environmental friendliness for single flights and all guided flights. Weather information and forecasts including severity of weather polygons could be shown in detail in the Meteorological Service display, connected to the radar screen if useful.

One key feature of the ICM working position is the team conference monitor. It contains several features of social interaction among the ICM and one or more team members (in this case two tactical controllers and one TMA controller). With option buttons the ICM selects a controller resp. the view of the tactical controllers' traffic situation. Whenever the traffic situation allows it, the ICM introduces a new training unit, i.e. a new approach procedure. As shown in Fig. 2 the ICM invites a selected controller via the team conference monitor to train a certain time based arrival approach by using so called Ghosts and TargetWindows. The ICM working position allows a supervised on-the-job training helping the tactical controller to become more familiar with new procedures while on duty instead of completing after-work training sessions in simulators which are timely demanding and costly. It is obvious, that the job of an ICM requires a well-established knowledge of air traffic management particularly of the future guidance methods as proposed by SESAR.

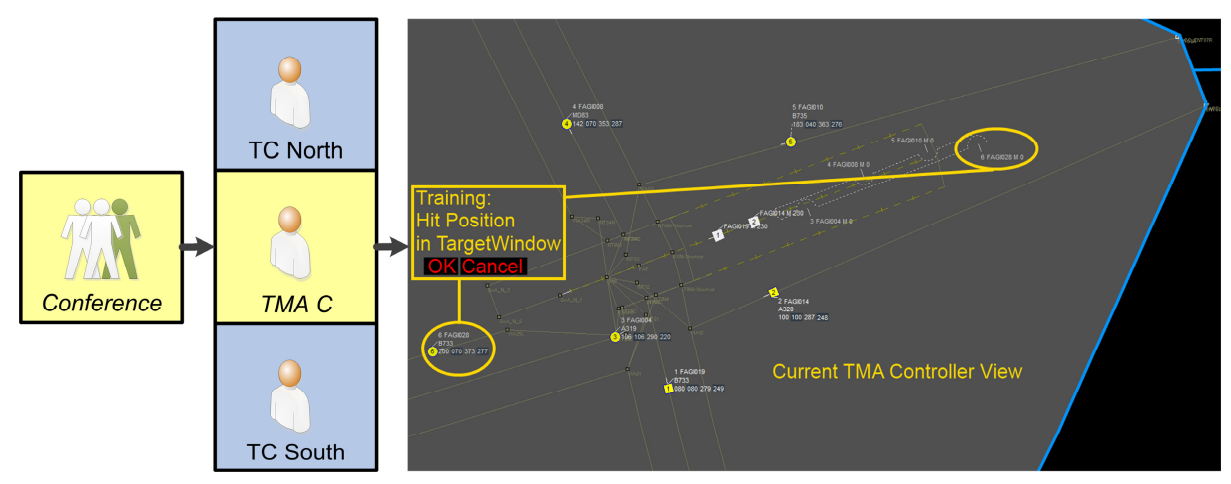

Fig. 2. ICM advises special on-the-job training to TMA controller via HMI

The described amount of information the ICM has to monitor, would definitely exceed the cognitive capacity of a tactical controller in situations under heavy workload. Information regarding the consolidation of conflicts, runway closures or the weather concerning one aircraft or airport can also be "given" to the operational controller by the ICM. The controller again may forward this given advice to a pilot via data link.

Three possible scenarios are explained in the following. If plan deviations occur or a long-term forecasted situation may lead to a conflict, the ICM may advise a conflict solution by changing altitudes and propose it to the most convenient controller. Another scenario refers to the wind direction and intensity. The ICM then advises several noise optimized routes to the operating controller. 
If the airline or the pilot wishes to change an arrival route or a procedure, the ICM would check if traffic and weather permits it. After checking the situation, the ICM passes this information to the operational controller. The integration of what-ifsolutions for the en-route area to check decisions before delegating to the next controller would be very helpful. All these mentioned points would probably not be handled as easy by a tactical or TMA controller without ICM support.

\subsection{Effects of Controller Roles on HMI}

The HMI view of tactical controllers will change with the introduction of new guidance steps and our proposed ICM role. One basic possibility is the marking of one aircraft in a general radar screen by any input device to gain more information. In the distance based display the minimum lateral separation around the aircraft can be shown. The vertical distance may be visualized via a three-dimensional view or by warning only if a separation miss occurs. The time based step includes all features of the former step, but shades them darker, because this information will be less frequently used due to pre-regulation. The time based display concentrates on time based separation resp. negotiated target times at different waypoints and marks. Controllers have to check, if all times calculated by a short term flight prediction out of the current flight status can be held. The trajectory based view mainly focuses on the conformance monitoring. Negotiated four-dimensional trajectories with various trajectory points are planned and compared with the real flown way. The information of former displays only appears very dark. The final performance based display shows individual capabilities of the chosen aircraft. It is possible to show the optimum, minimum and maximum time reaching next significant waypoints, rates of climbing, descending, accelerating and reducing, the arrival approach and current weight.

In addition a "channel" for important incoming messages of the ICM should be available. To design the transition as smooth as possible, the former flight strip access could be used. Traditionally paper strips with aircraft information are given to the controller when an aircraft is reaching the area of responsibility. These strips are used to inform and make notes on every single flight and are handed over to the next controller if needed. Some ANSPs already use digital flight strips appearing on the HMI when the aircraft is expected to enter the area of control soon. Important information on weather or critical events could also be noted on special information strips given by the ICM. If the controller accepts the information, he can decide whether to consider or to discard the data. The responsibility to guide air traffic lies within the sovereignty of controllers and therefore is expected to stay largely unchanged even with new roles and HMIs.

\section{$5 \quad$ Summary and Outlook}

Many different challenges in ATM will influence controller work in the future. Our proposed new position of an Information-and-Conflict-Manager (ICM) shall get along with information of the system wide information management network concept.

The position is also responsible for training of supervised operational controllers. Additional information and envisaged implementation of the three steps of controller 
guidance approaches deserve new long-lasting HMI solutions. Nevertheless, hardly any aspect of the challenges is handled in actual controller HMIs. The ICM working position and his tasks will support other controllers to avoid excessive workload. Training of new flight guidance concepts can be done on-the-job and help to successfully overcome the hurdles from one guidance approach step to the other. Besides that, it will reduce costs in simulator-based training.

The theoretical concept was developed due to user-centered design norms. In order to operationally use this concept, a controller evaluation and praxis test is needed. However, the user centered migration tolerant human computer interaction is a way towards future work environmental changes at controller HMIs.

\section{References}

1. Gregorova, M.: EUROCONTROL: EUROCONTROL Long-Term Forecast - Flight Movements 2010 - 2030 (2010),

http://www. eurocontrol.int/sites/default/files/content/ documents/official-documents/forecasts/long-term-forecast2010-2030.pdf

2. EUROCONTROL: System Wide Information Management (SWIM). SESAR factsheet. No. 01/2011 (2011)

3. Latron, P., McGregor, R., Geissel, M., Marsden, A.: En-route Multi Sector Planning Procedures - PHARE/EEC/PD3-3.1.3.2.5/SSR;01, further information of EUROCONTROL, Bruxelles (1997),

http: //www.eurocontrol.int/phare/public/standard_page/ MSP. html

4. Sorensen, C., Crook, I., Liang, D., Jehlen, R.: The Area Flow Multi-Sector Planner: A Fast-Time Study of MSP Coordination Activities. In: Eighth USA/Europe Air Traffic Management Research and Development Seminar (2009)

5. Graham, R., Marsden, A., Pichancourt, I., Dowling, F.: Controller Roles - Time to change. In: Third USA/Europe Air Traffic Management Research and Development Seminar (2000)

6. König, C., Hofmann, T., Bruder, R.: Application of the user-centered design process according ISO 9241-210 in air traffic control. Work 41, 167-174 (2012)

7. Rauterberg, M.: Usability Engineering Methods and Tools. User Centered Design. Technical University Eindhoven, Eindhoven (1998),

http://www.idemployee.id.tue.nl/g.w.m.rauterberg/

publications/UCD_Tutorial.pdf

8. Ferrara, G.: Evolving aircraft capabilities in the context of SESAR Concept (2010)

9. Celio, J., Smith, E.: Performance-based Air Traffic Management: Evaluating Operational Acceptability. In: Seventh USA/Europe Air Traffic Management Research and Development Seminar (2007)

10. Uebbing-Rumke, M., Temme, M.: Controller Aids for Integrating Negotiated Continuous Descent Approaches into Conventional Landing Traffic. In: Ninth USA/Europe Air Traffic Management Research and Development Seminar (2011)

11. Vales, S., Dupré, C., Gaspard-Boulinc, H., Conversy, S., Ollagnon, C., Peyruquéou, V., Viala, J.: MAMMI Phase3 - Exploring workspaces for Air Traffic Controllers in the scope of SESAR (2008)

12. EUROCONTROL: SWIM Pioneer (NOP B2B - Business to Business). SESAR factsheet. No. 08/2011 (2011)

13. EUROCONTROL SWIM homepage, http: / /www . eurocontrol. int/services/system-wide-informationmanagement-swim 